\title{
ERICA and WikiTalk
}

\author{
Divesh Lala $^{1}$, Graham Wilcock ${ }^{1,2}$, Kristiina Jokinen ${ }^{3}$ and Tatsuya Kawahara ${ }^{1}$ \\ ${ }^{1}$ Graduate School of Informatics, Kyoto University, Japan \\ ${ }^{2} \mathrm{CDM}$ Interact, Helsinki, Finland \\ ${ }^{3}$ Artificial Intelligence Research Center, AIST Tokyo Waterfront, Japan \\ lala@sap.ist.i.kyoto-u.ac.jp, graham.wilcock@cdminteract.com, \\ kristiina.jokinen@aist.go.jp, kawahara@i.kyoto-u.ac.jp
}

\begin{abstract}
The demo shows ERICA, a highly realistic female android robot, and WikiTalk, an application that helps robots to talk about thousands of topics using information from Wikipedia. The combination of ERICA and WikiTalk results in more natural and engaging human-robot conversations.
\end{abstract}

\section{Introduction}

The demo shows the results of combining a highly realistic android robot with an application that helps robots to talk fluently about thousands of topics using information from Wikipedia. The aim is to work towards a level of realism in spoken dialogue and associated non-verbal behaviours that enables more natural and more engaging conversations between humans and robots.

\subsection{ERICA}

ERICA is a female android that is being developed as a conversational robot with a variety of roles [Glas et al., 2016]. ERICA's appearance is highly realistic so her conversational behaviors should also be human-like. Her text-to-speech synthesized voice is trained on a real voice actress, allowing her to generate realistic sounding backchannels, laughs and fillers. ERICA captures the user's speech through a microphone array, so that the user is free to speak naturally without needing to hold a microphone. Speaker identification and human behavioral sensing is implemented through Kinect and a small camera.

We have already developed several functions for ERICA, including backchannels, statement response generation, engagement recognition and turn-taking models [Inoue et al., 2016; Lala et al., 2017; Lala et al., 2018; Inoue et al., 2018; Kawahara, 2018]. We have implemented ERICA in a number of roles such as an attentive listener and job interviewer. Using ERICA as an interface to Wikipedia is an additional role that we have implemented and will describe in Section 2.

\subsection{WikiTalk}

WikiTalk is an interactive spoken information access system that enables robots to talk fluently about thousands of topics using information from Wikipedia. The first implementation

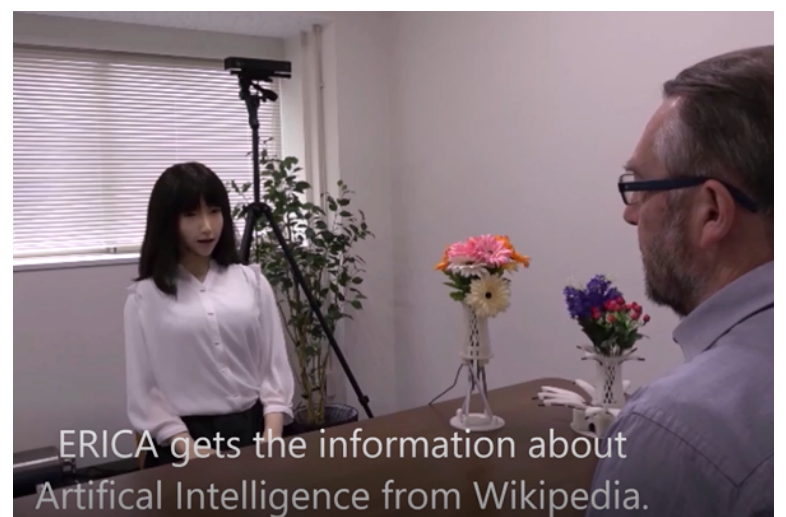

Figure 1: The demo video can be seen at https://www.youtube.com/ watch?v=Aq4Rfwrktr0.

with the Pyro toolkit for AI and robotics [Blank et al., 2006] demonstrated the capability for talking about whatever topics an individual user finds interesting, and for making smooth topic shifts to related topics using links to related Wikipedia articles [Jokinen and Wilcock, 2012; Wilcock, 2012].

The implementation of WikiTalk on NAO humanoid robots added non-verbal interaction capabilities including head nods, gesturing, face-tracking, and interrupting the robot by touching its head [Csapo et al., 2012]. The robot uses beat gestures (short vertical hand movements) to mark mentioned items as potential topic shifts [Meena et al., 2012].

The NAO robot speech recognition component allows the speech recognition vocabulary to be changed dynamically. WikiTalk exploited this by predicting likely next topics as the dialogue proceeds and adding them to the vocabulary to support smooth topic shifts [Jokinen and Wilcock, 2014].

WikiTalk is multilingual and can switch between English, Finnish and Japanese [Wilcock et al., 2016; Laxström et al., 2017]. Ongoing work aims at developing long-term relations with human partners, and adding Wikipedia-based listening to Wikipedia-based talking [Wilcock and Jokinen, 2019].

\section{ERICA and WikiTalk Implementation}

When using ERICA as an interface to Wikipedia, our aim is to make the interaction as natural as possible, to be compatible with her realistic appearance. The user should be able 
to speak to ERICA using natural language as they would to a human, rather than a robotic command-based interface. ERICA should recognize when to start searching for a Wikipedia page without explicit notification from the user.

There are some differences in the way speech recognition works in the implementations on NAO and ERICA. As the NAO speech components allow the recognition vocabulary to be changed dynamically, WikiTalk on NAO continually updates the vocabulary by predicting likely next topics as the dialogue proceeds. This allows even very rare or very new topics to be recognized.

ERICA uses Julius as the automatic speech recognition (ASR) system with both English and Japanese recognition capability [Lee and Kawahara, 2009]. In this paper we assume the language is English. Julius currently uses a static but very large speech recognition vocabulary. Although it is not updated dynamically with changing topics, the large vocabulary allows the user to choose a very wide range of topics.

With both NAO and ERICA, the user can ask about a Wikipedia topic they wish to hear about by simply saying for example I'd like to hear about [topic name]. NAO currently uses simple word-spotting with dynamically updated vocabularies, but ERICA uses a more flexible approach for extracting the Wikipedia topic, by applying natural language processing techniques to extract the desired topic from the input utterance as follows.

Firstly, since the ASR system outputs the result in lowercase, we use truecasing ${ }^{1}$ to estimate the correct capitalization. Then we find all the named entities and nouns in the utterance using the Python module spaCy ${ }^{2}$. Since there can be both named entities and nouns (both compound and simple) in the utterance, we use a heuristic to prioritize each candidate. Named entities are given preference over compound nouns, which are prioritized over single nouns. If there are multiple named entities or noun types, we choose the one that was said last in the utterance. For example, if the user says Well, you're a smart robot, so I want to know about philosophy the latest noun (philosophy) would be chosen by ERICA. With NAO, the word-spotting would depend on the dynamic vocabulary, so either robot or philosophy (or neither) would be recognized depending on the context.

Within Wikipedia there are many terms which could be related to multiple different topics, so disambiguation pages are required. For example, John Smith is the name of multiple famous people. Our system recognizes this disambiguation page and ERICA presents the list of candidate topics to the user. When the user hears the topic they wish to know about, they can say Yes to confirm.

With NAO speech components, turn-taking is very basic. Either the robot is listening or talking, but not both at the same time. To grab the turn while NAO is talking, WikiTalk supports interrupting by touching a sensor on the robot's head.

For ERICA, we use a more natural form of interaction. The user only has to start to talk and ERICA will capture their speech. The end of the user's turn is recognized after the user

\footnotetext{
${ }^{1}$ https://github.com/nreimers/truecaser

${ }^{2} \mathrm{https}: / /$ spacy.io/
}

is silent for a fixed period of time. ERICA signals that she has heard the user and is searching Wikipedia. When ERICA is speaking and the user wishes to hear about a related topic, the user can simply say that topic and if it is available, ERICA notifies the user and switches to that topic. If the user wishes to interrupt ERICA during her explanation, they can barge in and say Stop at any time. ERICA will stop speaking and apologize, allowing the user to choose a different topic.

On NAO, beat gestures are used to mark potential topic shifts, but synchronizing speech and gestures is difficult [Meena et al., 2012]. On ERICA, we tried marking potential topic shifts by changing the voice slightly, and this seemed to work quite well. This feature is optional and can be switched on or off. It is not used in the demo video.

\section{Description of the Demo Video}

The video ( $8.5 \mathrm{~min}$ ) can be seen at https://www.youtube.com/ watch?v=Aq4Rfwrktr0.

In the first part of the video (6 min), ERICA talks about classical languages and robots, and then talks more extensively about artificial intelligence. In the later part (2 min), ERICA talks to another person about android robots.

The video shows two ways that ERICA can change topics. In smooth topic shifts ERICA moves to a related topic. At the start of the video, she is talking about classical languages and mentions Greek and Latin. When the person asks her to talk about Greek, she makes a smooth topic shift to ancient Greek. There are many other Wikipedia articles with Greek in the title, but WikiTalk finds a link from classical languages to ancient Greek so that is the most relevant continuation.

To change to a completely unrelated topic, the person first asks ERICA to stop talking about Greek. She stops, shows surprise, and asks him to suggest another topic. He asks about robots, an unrelated topic. ERICA checks Wikipedia and starts talking about robots. When he asks to hear more about autonomous robots, she makes a smooth topic shift from robots to autonomous robots, and when he asks about artificial intelligence, she makes another smooth topic shift and starts talking about AI.

ERICA continues talking about the current topic if the person continues listening. In the video she keeps talking about AI for more than two minutes, until he asks her to switch to cognitive intelligence. As there is no Wikipedia article with that title, ERICA talks about intelligence.

In the later part, another person asks ERICA about android robots. She recognizes Android but not android robots. There are multiple articles about Android, so ERICA asks him to disambiguate from a list. As the first item is Android (robot), there is no need to go through the other alternatives. While talking about android robots, ERICA says that a robot with a female appearance can also be referred to as a gynoid.

\section{Acknowledgments}

This work was supported by JST ERATO Ishiguro Symbiotic Human-Robot Interaction program (Grant Number JPMJER1401), Japan. The third author acknowledges the support of the New Energy and Industrial Technology Development Organisation (NEDO), Japan. 


\section{References}

[Blank et al., 2006] Douglas Blank, Deepak Kumar, Lisa Meeden, and Holly Yanco. The Pyro toolkit for AI and robotics. AI Magazine, 27(1):39-50, 2006.

[Csapo et al., 2012] Adam Csapo, Emer Gilmartin, Jonathan Grizou, JingGuang Han, Raveesh Meena, Dimitra Anastasiou, Kristiina Jokinen, and Graham Wilcock. Multimodal conversational interaction with a humanoid robot. In Proceedings of 3rd IEEE International Conference on Cognitive Infocommunications (CogInfoCom 2012), pages 667672, Kosice, 2012.

[Glas et al., 2016] Dylan F. Glas, Takashi Minato, Carlos T. Ishi, Tatsuya Kawahara, and Hiroshi Ishiguro. ERICA: The ERATO Intelligent Conversational Android. In Proceedings of the IEEE International Symposium on Robot and Human Interactive Communication (RO-MAN 2016), pages 22-29, 2016.

[Inoue et al., 2016] Koji Inoue, Pierrick Milhorat, Divesh Lala, Tianyu Zhao, and Tatsuya Kawahara. Talking with ERICA, an autonomous android. In Proceedings of the 17th Annual Meeting of the Special Interest Group on Discourse and Dialogue, pages 212-215, Los Angeles, USA, 2016.

[Inoue et al., 2018] Koji Inoue, Divesh Lala, Katsuya Takanashi, and Tatsuya Kawahara. Engagement recognition in spoken dialogue via neural network by aggregating different annotators' models. In Proceedings of Interspeech 2018, pages 616-620, Hyderabad, India, 2018.

[Jokinen and Wilcock, 2012] Kristiina Jokinen and Graham Wilcock. Constructive interaction for talking about interesting topics. In Proceedings of Eighth International Conference on Language Resources and Evaluation (LREC 2012), Istanbul, 2012.

[Jokinen and Wilcock, 2014] Kristiina Jokinen and Graham Wilcock. Multimodal open-domain conversations with the Nao robot. In Joseph Mariani, Sophie Rosset, Martine Garnier-Rizet, and Laurence Devillers, editors, Natural Interaction with Robots, Knowbots and Smartphones: Putting Spoken Dialogue Systems into Practice, pages 213-224. Springer, 2014.

[Kawahara, 2018] Tatsuya Kawahara. Spoken dialogue system for a human-like conversational robot ERICA. In Ninth International Workshop on Spoken Dialogue Systems (IWSDS 2018), Singapore, 2018.

[Lala et al., 2017] Divesh Lala, Pierrick Milhorat, Koji Inoue, Masanari Ishida, Katsuya Takanashi, and Tatsuya Kawahara. Attentive listening system with backchanneling, response generation and flexible turn-taking. In Proceedings of the SIGDIAL 2017 Conference, pages 127136, Saarbrücken, Germany, 2017.

[Lala et al., 2018] Divesh Lala, Koji Inoue, and Tatsuya Kawahara. Evaluation of real-time deep learning turntaking models for multiple dialogue scenarios. In Proceedings of the 2018 Conference on International Conference on Multimodal Interaction, pages 78-86, Boulder, Colorado, 2018. ACM.
[Laxström et al., 2017] Niklas Laxström, Graham Wilcock, and Kristiina Jokinen. Internationalisation and localisation of spoken dialogue systems. In Kristiina Jokinen and Graham Wilcock, editors, Dialogues with Social Robots: Enablements, Analyses, and Evaluation, pages 207-219. Springer, 2017.

[Lee and Kawahara, 2009] Akinobu Lee and Tatsuya Kawahara. Recent development of open-source speech recognition engine Julius. In Proceedings of APSIPA ASC 2009: Asia-Pacific Signal and Information Processing Association, 2009 Annual Summit and Conference, pages 131$137,2009$.

[Meena et al., 2012] Raveesh Meena, Kristiina Jokinen, and Graham Wilcock. Integration of gestures and speech in human-robot interaction. In Proceedings of $3 \mathrm{rd}$ IEEE International Conference on Cognitive Infocommunications (CogInfoCom 2012), pages 673-678, Kosice, 2012.

[Wilcock and Jokinen, 2019] Graham Wilcock and Kristiina Jokinen. Towards increasing naturalness and flexibility in human-robot dialogue systems. In Tenth International Workshop on Spoken Dialogue Systems (IWSDS 2019), Siracusa, Italy, 2019.

[Wilcock et al., 2016] Graham Wilcock, Kristiina Jokinen, and Seiichi Yamamoto. What topic do you want to hear about? A bilingual talking robot using English and Japanese Wikipedias. In Proceedings of COLING 2016, the 26th International Conference on Computational Linguistics: System Demonstrations, Osaka, Japan, 2016.

[Wilcock, 2012] Graham Wilcock. WikiTalk: A spoken Wikipedia-based open-domain knowledge access system. In Proceedings of the COLING 2012 Workshop on Question Answering for Complex Domains, pages 57-69, Mumbai, India, 2012. 